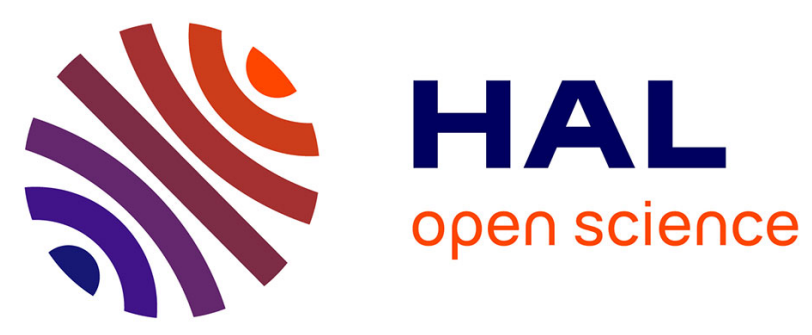

\title{
DÉTERMINATION GÉOMTRIQUE DE RÉPONSES IMPULSIONNELLES ÉCHOGRAPHIQUES SUR DES CIBLES IMMERGÉES DE FORME SIMPLE
}

\author{
R. Raillon, D. de Vadder, A. Lhémery
}

\section{- To cite this version:}

R. Raillon, D. de Vadder, A. Lhémery. DÉTERMINATION GÉOMTRIQUE DE RÉPONSES IMPULSIONNELLES ÉCHOGRAPHIQUES SUR DES CIBLES IMMERGÉES DE FORME SIMPLE. Journal de Physique IV Proceedings, 1992, 02 (C1), pp.C1-831-C1-834. 10.1051/jp4:19921181 . jpa00251143

HAL Id: jpa-00251143

https://hal.science/jpa-00251143

Submitted on 1 Jan 1992

HAL is a multi-disciplinary open access archive for the deposit and dissemination of scientific research documents, whether they are published or not. The documents may come from teaching and research institutions in France or abroad, or from public or private research centers.
L'archive ouverte pluridisciplinaire HAL, est destinée au dépôt et à la diffusion de documents scientifiques de niveau recherche, publiés ou non, émanant des établissements d'enseignement et de recherche français ou étrangers, des laboratoires publics ou privés. 


\title{
DETERMINATION GÉOMETRIQUE DE REPONSES IMPULSIONNELLES ÉCHOGRAPHIQUES SUR DES CIBLES IMMERGÉES DE FORME SIMPLE
}

\author{
R. RAILLON, D. de VADDER et A. LHÉMERY. \\ Laboratoire MSS/MAT, URA 850 C.N.R.S., Equipe Ultrasons, Ecole Centrale Paris, Grande Voie des \\ Vignes, F-92295 Châtenay-Malabry cedex, France
}

\begin{abstract}
We propose a simple interpretation of the mecanism of echo formation for targets of simple shape (spheres, cones and small tilted disks) placed far from a plane broad band transducer on its axis. This interpretation is based on the description of the radiated field in terms of plane and edge waves component. This permits to find easly the approximate shape and amplitude of the impulse response. The echo is then obtained by convolution of the latter with the excitation signal.

Résumé - Nous proposons une interprétation simple du mécanisme de formation des échos pour des billes cônes et petits disques inclinés placés assez loin sur l'axe d'un traducteur plan large bande. Cette interprétation utilisant la description du champ émis en termes d'onde géométrique et de bord permet de retrouver facilement la forme schématique des réponses impulsionnelles pour les cibles considérées. L'écho est ensuite obtenu par convolution avec le signal d'excitation.
\end{abstract}

\section{Introduction}

L'écho renvoyé par une cible tel que nous le voyons à l'oscilloscope est le résultat de la convolution d'une réponse impulsionnelle et d'un signal d'entrée que l'on peut obtenir expérimentalement [1]. La forme de la réponse impulsionnelle qui est l'écho renvoyé par la cible pour un traducteur émettant un dirac de vitesse particulaire est plus simple à interpréter que celle des échos qui dépendent (par la convolution) de la forme de la dépendance temporelle de la source. C'est donc la réponse impulsionnelle que nous étudions avant de calculer l'écho par convolution.

\section{Réponse impulsionnelle pour une cible sur l'axe du traducteur.}

Un traducteur plan, piston, uniforme, immergé dans un fluide et excité par un dirac de vitesse particulaire émet une onde géométrique de front plan et étendue à l'ensemble de sa surface et une onde de bord de forme toroïdale issue des points de la périphérie [2]. Ainsi, en un point $M$ à une distance ' $z$ ' sur l'axe du traducteur on observe un dirac de surpression à l'instant $\operatorname{tg}=z / c$ qui correspond à l'arrivée de l'onde géométrique puis un dirac de dépression à l'instant $t \mathrm{~b}=\left(\mathrm{z}^{2}+\mathrm{a}^{2}\right)^{1 / 2} / \mathrm{c}$ qui correspond à l'arrivée de l'onde de bord. ('a' rayon du traducteur et 'c' célérité du son).

Si le point $\mathrm{M}$ est désaxé, le passage de l'onde géométrique crée toujours un dirac à l'instant tg mais la contribution des points du bord du traducteur est étalée dans le temps car ils ne sont plus équidistants de $M$.

En émission réception une cible ponctuelle placée sur l'axe du traducteur a une réponse impulsionnelle composée de trois diracs sous la forme

$$
\begin{gathered}
H(t)=\left(\rho_{0} c\right)^{2}\left[\delta\left(t-t_{0}(z)\right)-2 \delta\left(t-t_{1}(z)\right)+\delta\left(t-t_{2}(z)\right)\right] \\
t_{0}(z)=2 z / c, t_{1}(z)=\left(\left(a^{2}+z^{2}\right)^{1 / 2}+z\right) / c \text { et } t_{2}(z)=2\left(\left(a^{2}+z^{2}\right)^{1 / 2}\right) / c
\end{gathered}
$$

Les trois instants correspondent à l'arrivée de l'onde géométrique (resp. de bord) diffractée par la cible et reçue par le centre puis le bord du traducteur.

Un désaxement de la cible entraîne un étalement des deux dernières contributions autour des instants $t_{1}$ et $t_{2}$. Quand la distance augmente les différentes contributions se rapprochent et la réponse impulsionnelle se concentre dans le temps.

La forme générale de la réponse impulsionnelle d'une cible quelconque [3], sous l'hypothèse d'une impédance infinie par rapport au milieu se simplifie en [4] 


$$
H(t)=\iint_{s} \frac{\cos \theta_{s}}{\rho_{0} c} h^{\partial t}\left(r_{s}, t\right) * h^{\partial t}\left(r_{s}, t\right) d S_{S}
$$

$r_{s}$ repère un élément de surface $d S_{S}$ de la cible, $\theta_{S}$ est l'angle que fait la normale à l'élément de surface avec l'axe du traducteur, $\rho_{0}$ et $\mathrm{c}$ sont la masse volumique et la célérité, $t$ le temps. $h^{\partial t}\left(r_{s}, t\right)$ est la réponse impulsionnelle en émission pour la pression au point $M\left(r_{s}\right)[3] . H^{\partial t}\left(r_{s}, t\right)=h^{\partial t}\left(r_{s}, t\right) * h^{\partial t}\left(r_{s}, t\right)$ est la réponse impulsionnelle en émission réception pour une cible ponctuelle placée au point $\mathbf{M}\left(\mathbf{r}_{\mathrm{s}}\right)$.

Nous faisons l'hypothèse suivante:

$$
\begin{gathered}
H^{\partial t}(r, z, t) \approx \operatorname{RIP}^{\partial t}\left(0, z_{0}, t\right) * \delta\left(t-\frac{2 z}{c}\right)=\operatorname{RIP}^{\partial t}\left(0, z_{0}, t-\frac{2 z}{c}\right) \\
\operatorname{RIP}^{\partial t}\left(0, z_{0}, t\right)=\left(\rho_{0} c\right)^{2}\left[\delta(t)-2 \delta\left(t-\left(t_{1}\left(z_{0}\right)-t_{0}\left(z_{0}\right)\right)+\delta\left(t-\left(t_{2}\left(z_{0}\right)-t_{0}\left(z_{0}\right)\right)\right)\right]\right.
\end{gathered}
$$

$t_{0}(z), t_{1}(z), t_{2}(z)$ sont définis en (2) et $z_{0}$ est la distance la plus courte entre la cible et le traducteur. La fonction $R I P(t)\left(0, z_{0}, t\right)$ est différente de $H^{\partial t}\left(0, z_{0}, t\right)$ : pour $H^{\partial t}$ la valeur de $t$ fixe le début de la réponse impulsionnelle par rapport à une origine des temps donnée alors que pour RIPðt la réponse débute à l'instant 0 . La convolution avec $\delta(t-2 z / c)$ positionnera l'ensemble de la réponse dans le temps. RIP ${ }^{\partial t}\left(0, z_{0}, t\right)$ est donc la réponse impulsionnelle ramenée à l'origine des temps pour une cible ponctuelle sur l'axe en $z_{0}$. Cette approximation (4) revient à calculer l'amplitude de l'écho en considérant que tous les points de la cible sont sur l'axe à la distance $\mathrm{z}_{0}$ du traducteur. La convolution avec le $\delta$ traduit le retard de la contribution de chaque point en fonction de la distance $\mathrm{z}$ de ce point au traducteur. Cela équivaut au calcul exact fait pour la phase en mono-fréquentiel. On a donc avec cette hypothèse et en posant $d S_{S}=A(z) d z$ :

$$
H(t)=\frac{1}{\rho_{0} c} \int_{z} R P^{\partial t}\left(0, z_{0}, t-\frac{2 z}{c}\right) \cos \theta_{s} A(z) d z
$$

en posant $2 z / c=t^{\prime}$ on obtient finalement:

$$
H(t)=\frac{1}{\rho_{0} c} \int_{t^{\prime}} R I P^{\partial t}\left(0, z_{0}, t-t^{\prime}\right) \cos \theta_{s} \frac{c}{2} A\left(\frac{c t^{\prime}}{2}\right) d t^{\prime}=\frac{1}{4 \rho_{0}} \operatorname{RIP}^{\partial t}\left(0, z_{0}, t\right) * P R(t)
$$

avec $P R(t)=\cos \theta_{s} A(c t / 2)$. Par le changement de variable inverse on définit $P R(z)=A(z) \cos \theta_{s} \operatorname{connu}$ dans la littérature comme la densité de surface projetée [6]. L'équation (7) qui est à rapprocher des résultats de [6] exprime donc simplement la réponse impulsionnelle comme le produit de convolution de trois diracs et de la densité de surface projetée de la cible. PR(z), connu analytiquement pour des cibles de forme simple, est représenté (fig 1) pour des cône, bille et disque incliné à partir des expressions de [5,6]:

- cône d'angle au sommet $2 \varepsilon$ : $P R(z)$ vaut $2 \pi\left(z-z_{0}\right) \tan ^{2} \varepsilon$ pour $z_{0}<=z<=z_{1}$ et 0 ailleurs. ( $z_{0}$ et $z_{1}$, cf fig 1$)$.

- bille de rayon $\mathrm{R}$ : $\operatorname{PR}(z)$ vaut $2 \pi\left(R-\left(z-z_{0}\right)\right)$ pour $z_{0}<=z<=z_{1}$ et 0 ailleurs.

- disque incliné de $\phi$ par rapport à l'axe du traducteur:

$P R(z)$ vaut $2\left(z_{0}-z_{1}\right)\left(L-L^{2}\right)^{1 / 2} \cot \phi / \sin \phi$ pour $z_{0}<=z<=z_{1}$ et 0 ailleurs avec $\mathrm{L}=\left(\left(\mathrm{z}-\mathrm{z}_{0}\right) /\left(\mathrm{z}_{1}-\mathrm{z}_{0}\right)\right)$.
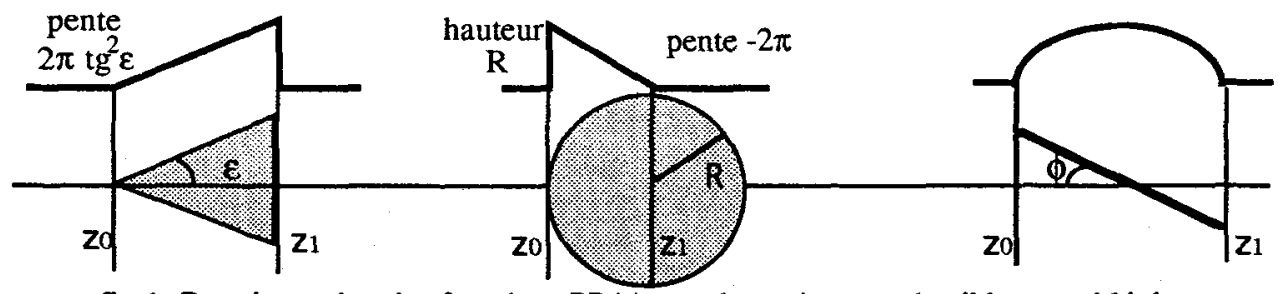

fig 1- Représentation des fonctions PR(z) pour les trois types de cibles considérées

- Représentation of the function PR(z) for targets of different shapes.'

Les approximations de l'équation (4) sont applicables aux petites cibles (car r voisin de 0 ) mais aussi aux billes et cônes plus grands s'ils sont assez loin sur l'axe du traducteur comme nous le verrons plus loin. 


\section{Interprétation de la réponse impulsionnelle approchée et résultats.}

Les résultats précédents peuvent être interprétés simplement: on a supposé la cible assez loin du traducteur pour pouvoir considérer l'onde géométrique et l'onde de bord comme des ondes planes de front perpendiculaire à l'axe du traducteur et dont la dépendance temporelle est un dirac positif ou négatif respectivement. La contribution à la réponse impulsionnelle d'une telle onde plane est proportionnelle à la quantité de surface projetée de la cible perpendiculairement à l'axe du traducteur. Elle varie donc avec le temps comme la fonction $\mathrm{PR}(\mathrm{t})$. En fait, comme pour la cible ponctuelle, chaque type d'onde après diffraction contribue deux fois à la réponse (retour de l'onde au centre puis au bord du traducteur [2]). La seconde contribution de l'onde géométrique et la première de l'onde de bord sont simultanées. Pour retrouver la contribution globale il faut donc sommer trois contributions décalées dans le temps, chacune ayant la forme de $P R(t)$, affectées respectivement des coefficients $1,-2,1$.

Une illustration de ce qui précéde est présentée pour la bille (fig2). (On procéderait de la même façon pour les cônes et disques).

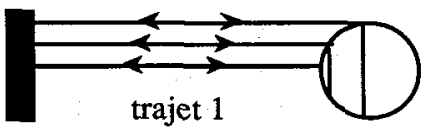

centre traducteur-cible-centre traducteur

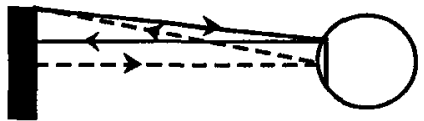

trajet 2 double

centre traducteur-cible-bord traducteur bord traducteur- cible-centre traducteur

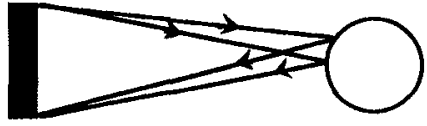

trajet 3

bord traducteur-cible-bord traducteur contribution pour chaque trajet contribution totale
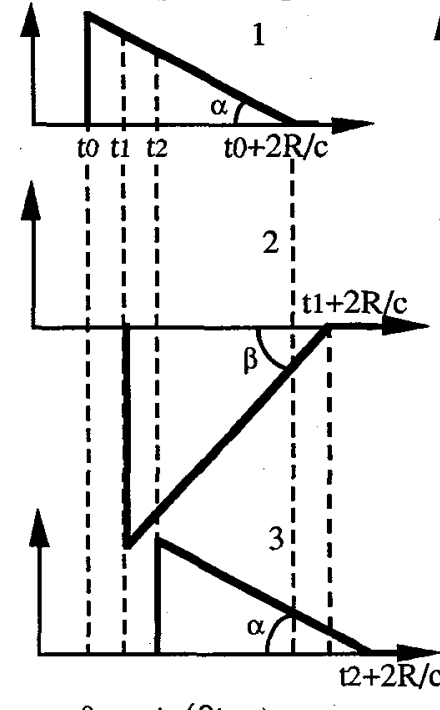

$\beta=\operatorname{arctg}(2 \operatorname{tg} \alpha)$

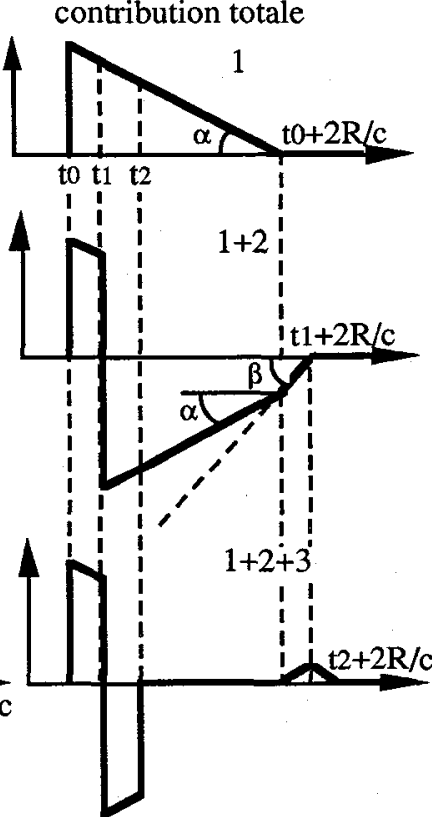

Fig 2 - Interprétation de la formation d'écho renvoyés par une bille placée sur l'axe d'un traducteur plan. - Interpretation of the echo formation for a ball target on the axis of a plane transducer.

Pour les billes et cônes on distingue deux parties dans les réponses impulsionnelles (fig 4 bille $2 \mathrm{~mm} \emptyset$ ). La deuxième partie est due à la périphérie du cône ou à l'ombre géométrique de la bille. La première partie est due à une zone de la cible (zone de diffraction) qui est en fait très petite autour du point de la cible situé sur l'axe en $z_{0}$ ce qui justifie les approximations $r=0$ et $z=z_{0}$ même pour des grandes cibles .

En effet cette première partie de la réponse impulsionnelle est nulle à partir de l'instant $t_{2}$ (fig2). Les points de la cible les plus éloignés du traducteur qui contribuent à l'écho à cet instant sont en $\mathrm{z}=\mathrm{ct}_{2} / 2$ et leur position définit sur la cible la limite de la zone de diffraction. Pour une cible donnée, la taille de la zone de diffraction est d'autant plus petite que l'intervalle de temps $\left(t_{2}-t_{0}\right)(\$ 1 b)$ est petit, cela est d'autant mieux vérifié que la distance cible traducteur est grande et le diamètre du traducteur petit .

Pour les petites cibles billes ou cônes, les deux parties de la réponse impulsionnelle sont assez proches pour former un seul écho après convolution avec le signal d'entrée, tandis que pour des cibles plus grandes les deux parties restent séparées temporellement même au niveau de l'écho. Dans ce dernier cas la deuxième partie de la contribution se retrouve mal avec le modèle simple car les points qui y contribuent sont très désaxés, et seule la première partie des échos qui est la plus caractéristique de la cible a été représentée.

Pour des disques inclinés le signal revient d'une petite zone autour des bords du disque. L'approximation n'est valable que si les bords sont près de l'axe c'est à dire pour des petits disques.

Des réponses impulsionnelles et des formes d'échos obtenus avec ce modèle simple, comparées avec des résultats de simulations précises (programmes de A Lhémery décrits ref 1 ) et des résultats expérimentaux de C Gatcombe [7] montrent un bon accord (fig 3 et 4). 

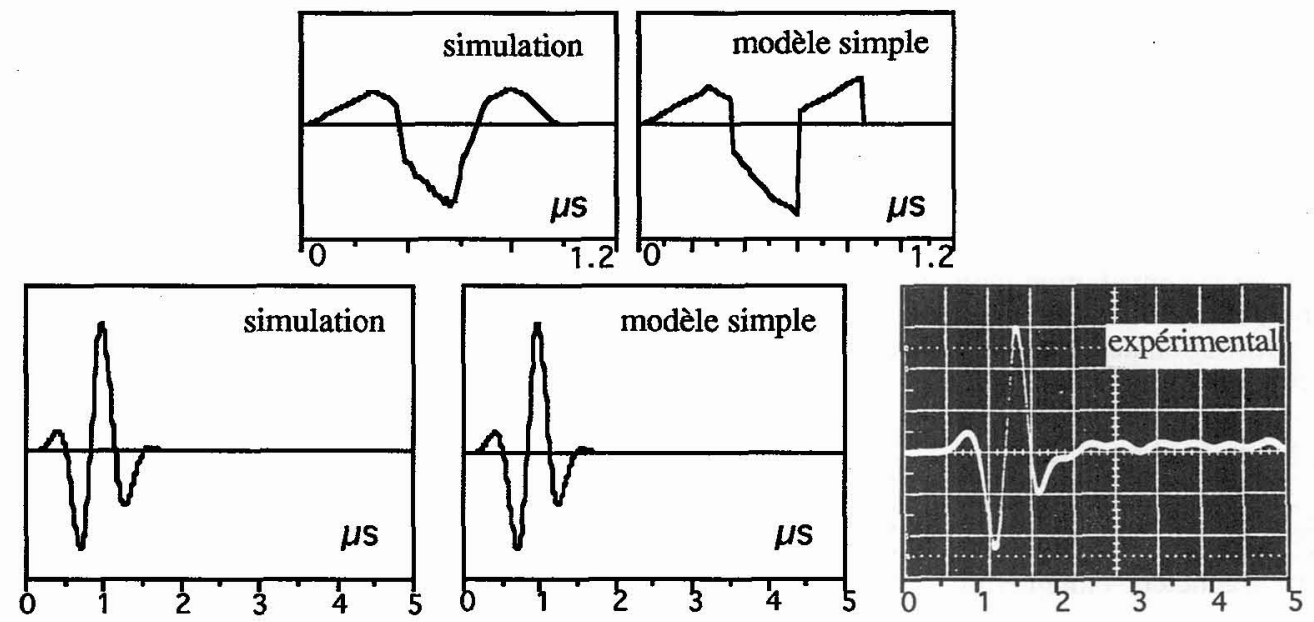

Fig 3 - Impulse response (top) and echos (bottom) for a conical target on axis,

$\left(\varepsilon=75^{\circ}, 2 \mathrm{~mm} \emptyset\right.$ maximal, distance $=120 \mathrm{~mm}$, plane transducer $19 \mathrm{~mm} \emptyset$, arbitrary units).
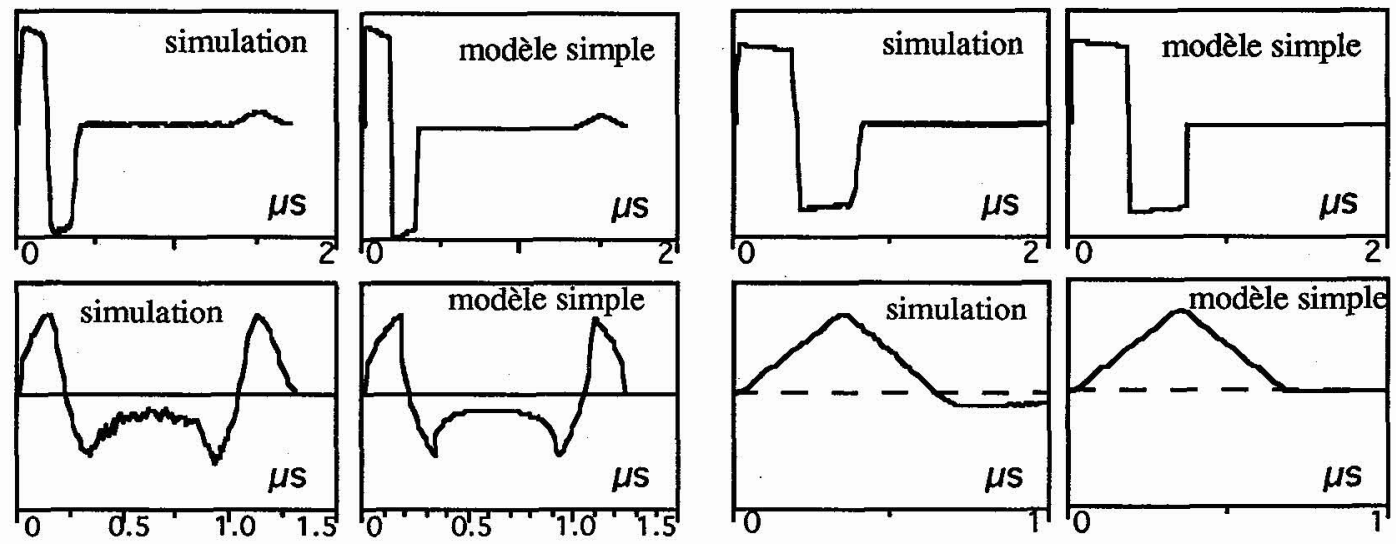

Fig 4 - Impulse response and echos for different targets on axis, plane transducer $20 \mathrm{~mm} \emptyset$,arbitrary units.

Top: a) balls $2 \mathrm{~mm} \emptyset$, distance $\mathrm{z}=200 \mathrm{~mm}$

Bottom: a) disk $2 \mathrm{~mm} \varnothing, 20^{\circ}$ tilted, $z=200 \mathrm{~mm}$

b) balls $8 \mathrm{~mm} \emptyset, z=90 \mathrm{~mm}$

b) conical target $\varepsilon=75^{\circ}, 20 \mathrm{~mm} \emptyset$ maximal, $z=100 \mathrm{~mm}$

\section{Conclusion}

Ce modèle permet de calculer la forme de certaines réponses impulsionnelles par une construction géométrique simple (surtout pour les cônes et billes). Quantitativement, les résultats obtenus concordent bien avec ceux des simulations et expériences (résultats non présentés ici). L'interprétation physique permet de prévoir facilement l'effet de différents paramètres géométriques (distance cible traducteur, dimensions du traducteur et de la cible) sur la forme de la réponse impulsionnelle ainsi que les limites de validité des approximations faites.

[1] A. Lhémery, thèse université du Maine, (1990).

[2] J.P. Weight and A.J.Hayman, J. Acoust. Soc. Am. 63 (1978), p 396-404.

[3] A. Lhémery, J. Acoust. Soc. Am. 90 (1991), p 2799-2807.

[4] A. Lhémery, proceeding du 2ième $\overline{C F}$ A, Arcachon 1992

[5] A.Freedman, Acustica, 12, (1962), p 61-70

[6] N.F. Haines, D.B.Langston, J. Acoust. Soc. Am. 67 (1980), p 1443-1454.

[7] C.Gatcombe, PhD thesis, The City University, (London United Kingdom) (1990). 